\title{
Междолинные процессы релаксации состояний мелких доноров в германии
}

\author{
(C) В.В. Цыпленков, В.Н. Шастин
}

Институт фризики микроструктур Российской академии наук, 607680 Нижний Новгород, Россия

E-mail: Tsyplenkov1@yandex.ru

Поступила в Редакцию 12 апреля 2021 г.

В окончательной редакции 19 апреля 2021 г.

Принята к публикации 19 апреля 2021 г.

\begin{abstract}
Проведен анализ роли междолинных процессов электрон-фононного взаимодействия в релаксации возбужденных мелких доноров мышьяка в германии. Вычислены темпы внутрицентровых междолинных переходов при излучении ТА фононов в германии в зависимости от одноосной деформации сжатия в кристаллографическом направлении $\{111\}$. Показано, что междолинные переходы с излучением фононов в основное состояние донора могут играть существенную роль в релаксации возбужденных примесей лишь при одноосной деформации кристалла, так как при нулевой деформации нет точных резонансов примесных переходов с энергией междолинных фононов. Также имеют место переходы из высоковозбужденных состояний, лежащих в узком пояске энергий $(\sim 0.5 \mathrm{мэB})$ у самого дна зоны проводимости в первое возбужденное состояние $1 s^{(3)}\left(\Gamma_{5}\right)$ (в деформированном Ge в $1 s^{(3)}\left(\Gamma_{3}\right)$ ), средний темп которых оценивается в $0.3 \cdot 10^{9} \mathrm{c}^{-1}$.
\end{abstract}

Ключевые слова: германий, мелкие доноры, электрон-фононное взаимодействие, междолинные переходы.

DOI: $10.21883 /$ FTP.2021.09.51298.28

\section{1. Введение}

В настоящее время изучение квантовых оптически управляемых когерентных состояний различных систем и создания на их основе принципиально новых полупроводниковых устройств, сопряженных с развитой кремниевой и германиевой технологией является актуальной задачей. Основой для таких устройств могут являться кулоновские центры в полупроводниках [1-6]. Основной интерес связан с разработкой элементов квантово-информационных систем, включающих устройства и методы считывания и записи информации, что предполагает использование различных подходов, основанных на свойствах квантово-механических систем, в частности на возможности манипуляции спиновыми и орбитальными состояниями. Возможность использования кулоновских центров для создания такого рода устройств во многом сводится к временам жизни возбужденных состояний рассматриваемых центров (продольное время релаксации) и времени существования когерентности осцилляций диполей, связанных с центрами, образующихся при резонансном внутрицентровом оптическом возбуждении среды. Поэтому исследование релаксационных процессов состояний доноров является в этом контексте актуальной задачей. Поскольку проведенные с использованием излучения Новосибирского лазера на свободных электронах (ЛСЭ NovoFEL) методом пробного импульса (pump-probe) измерения времен релаксации неравновесных состояний донорных центров в германии при внутрицентровом возбуждении $[7,8]$ показывают более длительные времена релаксации по сравнению со случаем кремния, то кристаллический германий с мелкими примесными центрами вызывает особенный интерес.

Такие измерения в кристалле германия, легированного донорами мышьяка и сурьмы (As, Sb) [7,8], вопреки ожиданиям, дают основания полагать, что существуют процессы релаксации состояний доноров, связанные с междолинными переходами при излучении акустических фононов. Согласно эксперименту, длительности рuтpprobe откликов при возбуждении высоколежащих по энергии состояний (выше $2 p_{ \pm}$) меньше, чем при возбуждении нижележащих. Это обстоятельство говорит о существовании прямых переходов из возбужденного состояния в состояния $1 s$, минуя промежуточные уровни, так как длительность отклика определяется общим временем возвращения носителей заряда в основное состояние донора после возбуждения. Полагается, что эти прямые каналы релаксации могут быть связаны с междолинными переходами при излучении акустических фононов. Волновые вектора междолиных фононов в германии лежат в окрестности X точки зоны Бриллюэна, а энергии этих фононов сосредоточены в довольно узком энергетическом диапазоне ( 9.7-10 мэВ). Из-за этого роль междолинных переходов в релаксации состояний мелких доноров в германии не очевидна, так как в недеформированном кристалле нет явных резонансов между энергиями внутрицентровых переходов и междолинных фононов. Однако существует возможность междолинных переходов из высоковозбужденных состояний, уровни которых лежат в узком диапазоне энергий под самым дном зоны проводимости, в состояния $1 s$ триплета, и переходов из более низколежащих уровней в основное состояние донора при отсутствии точного резонанса. 


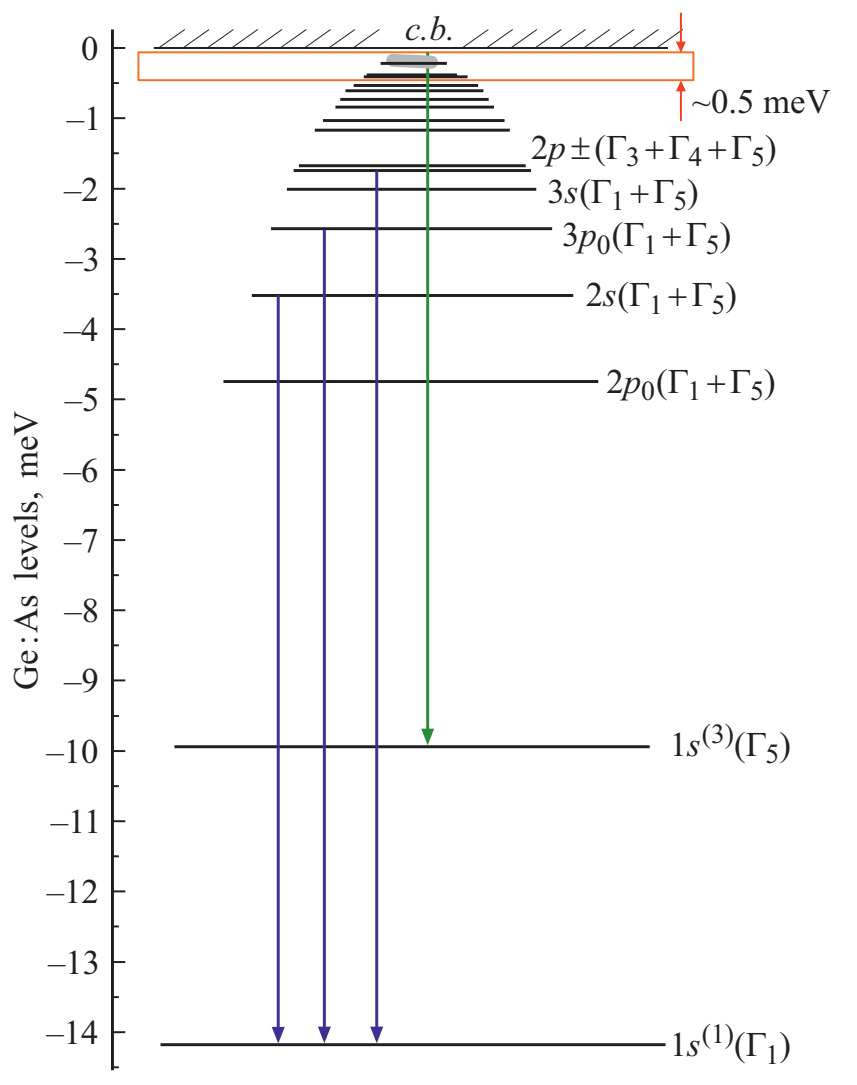

Рис. 1. Схема основных внутрицентровых междолинных переходов в $\mathrm{Ge}: \mathrm{As}$. Зеленой стрелкой показаны переходы из группы состояний, уровни которых лежат в узком пояске энергий под самым дном зоны проводимости. (Цветной вариант рисунка представлен в электронной версии статьи).

Схема внутрицентровых междолинных переходов в недеформированном Ge: As представлена на рис. 1.

\section{2. Теоретическая модель и результаты}

Волновые функции состояний донора выбирались в рамках метода эффективных масс [9]:

$$
\Psi(\mathbf{r})=\sum_{(i=1)}^{4} \alpha_{i} F(\mathbf{r}) \varphi_{i}(k, \mathbf{r}),
$$

где суммирование проводится по всем четырем долинам зоны проводимости германия, коэффициенты $\alpha_{i}$ определяют весовой вклад $i$-й долины в состояние донора, $F(\mathbf{r})$ - огибающая волновой функции, определяемая гамильтонианом, в который не входит периодический потенциал решетки кристалла, а вместо массы электрона входят его эффективные массы, $\varphi_{i}(\mathbf{k}, \mathbf{r})-$ волновая функция Блоха на дне $i$-й долины. Схема долин зоны проводимости германия и междолинных переходов показана на вставке рис. 2.

В качестве огибающих волновых функций использовались пробные функции [10], представляющие собой водородоподобные волновые функции, в которых анизотропия эффективной массы кристалла учитывалась путем следующей замены модуля вектора r:

$$
\frac{r}{a_{\mathrm{B}}} \rightarrow \sqrt{\frac{x^{2}+y^{2}}{a^{2}}+\frac{z^{2}}{b^{2}}} .
$$

Здесь $a_{\mathrm{B}}-$ боровский радиус, $a$ и $b-$ варьируемые параметры, определяющие локализацию волновой функции в пространстве. Явный вид огибающих волновых функций в пространстве волновых векторов в системе координат, в которой ось $z$ направлена вдоль оси долины, представлен в опубликованной ранее работе авторов [11]. Для состояний с $n>1(n-$ главное квантовое число) параметры $a$ и $b$ определены из вариационной процедуры минимизации энергии состояния с гамильтонианом эффективных масс и кулоновским примесным потенциалом [10]. Основное состояние доноpa $1 s(\mathrm{~A})$ сильно подвержено действию короткодействующего потенциала (потенциала центральной ячейки), действие которого учитывалось лишь через локализацию состояния, т. е. через параметры $a$ и $b$. Значения этих параметров для состояния $1 s(\mathrm{~A})$ вычислялись, используя квазиклассический подход определения асимптотического поведения функции при больших $r$, с опорой на экспериментальное значение энергии $(E)$ этого состояния, который сводится к следующим формулам [12]:

$$
a=\sqrt{\frac{\hbar^{2}}{2 m_{t} E}} ; \quad b=\sqrt{\frac{\hbar^{2}}{2 m_{l} E}},
$$

где $m_{l}$ и $m_{t}$ - продольная и поперечная эффективные массы зоны проводимости германия.

При расчете матричного элемента взаимодействия с коротковолновыми фононами края зоны Бриллюэна использовался такой же подход, как и в случае кристалла кремния [13,14]. В этом подходе матричный элемент рассчитывается с использованием усредненной характеристики кристалла - междолинного деформационного потенциала, определяющего средний матричный элемент междолинного перехода между состояниями свободного электрона. Значения деформационных потенциалов взяты из работы [15].

Особую сложность представляет суммирование по конечным состояниям системы - волновым векторам излучаемых фононов, так как это требует детального знания формы изоэнергетических поверхностей для ТА ветвей фононного спектра в окрестности точки X зоны Бриллюэна для различных значений энергии фононов. В литературе эти данные найти не удалось. Формы этих поверхностей отличаются для двух ТА веток и могут качественно меняться при небольшом изменении энергии фонона. В расчетах положено, что взаимодействие по аналогии с кремнием главным образом осуществляется с верхней ТА ветвью. Анализируя закон дисперсии фононов в различных направлениях $(\Gamma-\mathrm{X}-\mathrm{K}$, 


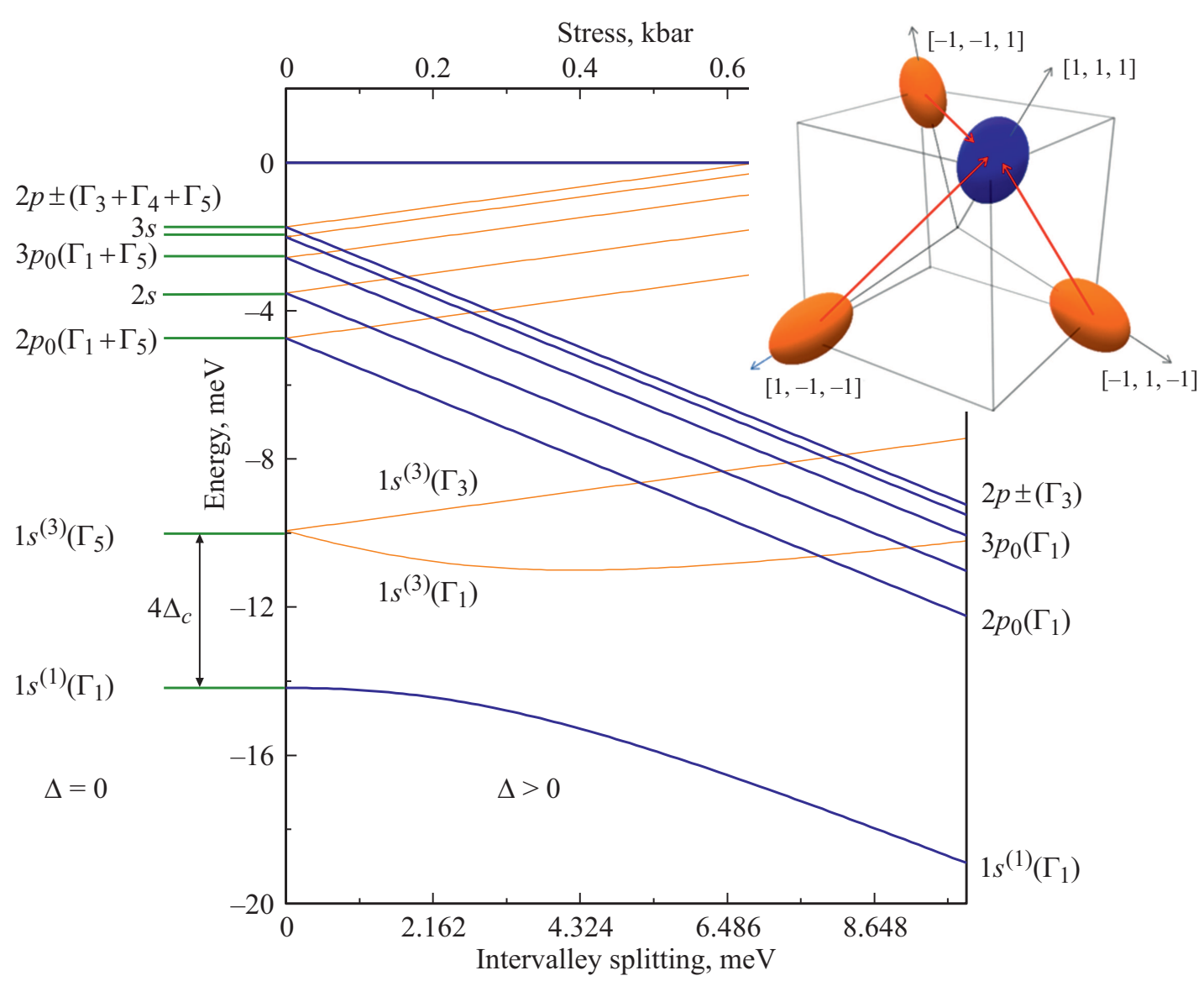

Рис. 2. Схема уровней донора мышьяка в германии в зависимости от деформации кристалла в кристаллографическом направлении [111]. На вставке показана схема долин зоны проводимости германия (центры долин расположены в точках L зоны Бриллюэна кристалла). Цвета долин соответствуют цветам линий уровней. Красными стрелками показаны междолинные переходы, серыми направления ориентации долин. (Цветной вариант рисунка представлен в электронной версии статьи).

$\mathrm{X}-\mathrm{W}, \mathrm{L}-\mathrm{X})[16]$, можно предположить, что в непосредственной окрестности точки $\mathrm{X}$ изоэнергетическая поверхность фононов для верхней ТА ветви приближенно может быть аппроксимирована цилиндром, вытянутым вдоль направления $\{100\}$ и симметричным относительно этого направления, в котором энергия фононов квадратичным образом зависит от радиуса цилиндра.

Одноосная деформация кристалла приводит к расщеплению энергетических уровней и частичному снятию вырождения примесных состояний, что напрямую влияет на их темпы релаксации. Зависимости от приложенного давления энергий уровней донора мышьяка в германии представлены на рис. $2[17,18]$. Распределение состояний мелких доноров по долинам зоны проводимости германия (коэффициенты $\alpha_{i}$ в формуле (1)) в зависимости от междолинного расщепления $(\Delta)$, вызванного одноосным сжатием кристалла в направлении [111], дается выражениями в таблице [17].

Величины одноосного сжатия кристалла, рассматриваемые в данной работе ( $\sim$ кбар), слишком малы для сколь-нибудь существенного изменения параметра решетки кристалла, поэтому изменение фононного спектра при таких значениях деформации пренебрежимо мало. В настоящей работе полагается, что влияние деформации на скорости релаксации состояний доноров происходит лишь через изменение спектра примесного центра и распределения состояний донора по долинам зоны проводимости в кристалле германия.

Результаты расчетов, представленные на рис. 3, показывают, что междолинные переходы в основное состояние донора $1 s^{(1)}\left(\Gamma_{1}\right)$ из возбужденных состояний донора, связанных с нижними долинами зоны проводимости, имеют место лишь при одноосной деформации кристалла. Поскольку рассматриваются переходы только между состояниями, связанными с нижними долинами, то матричный элемент перехода из возбужденных в основное состояние пропорционален вкладу верхних долин в основное состояние (коэффициенту $a^{-}$(см. таблицу)), который быстро убывает с ростом давления.

Темпы междолинных переходов в рамках используемой модели отличны от нуля лишь в очень узких диапазонах величин прикладываемого к кристаллу давления, что связано с особенностями фононного закона дисперсии в кристалле германия. При деформации кристалла кривые, достигая своего максимума, при точном резонансе обрываются, что связано с тем, что энергия 
Зависимость коэффициентов $\alpha_{i}$, определяющих долинные вклады в волновую функцию состояния донора, от междолинного расщепления

\begin{tabular}{c|c}
\hline & $\left(\alpha_{1} \alpha_{2} \alpha_{3} \alpha_{4}\right)$ \\
\hline $1 s^{(1)}\left(\Gamma_{1}\right)$ & $\alpha_{1}=\frac{1}{\sqrt{2}} a^{+} ; \quad \alpha_{2}=\alpha_{3}=\alpha_{4}=\frac{1}{\sqrt{6}} a^{-}$ \\
\hline \multirow{2}{*}{$1 s^{(3)}\left(\Gamma_{3}\right)$} & $\alpha_{1}=0 ; \quad \alpha_{2}=\frac{1}{\sqrt{2}} ; \quad \alpha_{3}=-\frac{1}{\sqrt{2}} ; \quad \alpha_{4}=0$ \\
& $\alpha_{1}=0 ; \quad \alpha_{2}=\frac{1}{\sqrt{2}} ; \quad \alpha_{3}=-\frac{1}{\sqrt{2}} ; \quad \alpha_{4}=0$ \\
\hline $1 s^{(3)}\left(\Gamma_{1}\right)$ & $\alpha_{1}=\frac{1}{\sqrt{2}} a^{-} ; \quad \alpha_{2}=\alpha_{3}=\alpha_{4}=a^{+}$ \\
\hline & $a^{ \pm}=\sqrt{1 \pm \frac{x-1 / 2}{\sqrt{1-x+x^{2}}}}, \quad x=\frac{\Delta}{\Delta_{c}}$
\end{tabular}

Примечание. $\Delta_{c}-$ химический сдвиг, определяемый как четверть энергетического зазора между основным состоянием и триплетным состоянием $1 s$.

внутрицентрового перехода становится ниже энергии фонона в X точке зоны Бриллюэна, и просто исчезают фононы, при излучении которых может осуществляться междолинный переход. Такая специфическая форма кривых является следствием неточности аппроксимации формы изоэнергетических поверхностей фононов. Согласно модели, их форма в области $k$-пространства, очень близкой к точке $\mathrm{X}$, является цилиндром с очень маленьким радиусом, что может не соответствовать действительности. Однако полученные результаты дают представление о величине скоростей междолинных внутрицентровых переходов и характере зависимости этих скоростей от деформации кристалла.

Переходы в состояние триплета $1 s^{(3)}\left(\Gamma_{5}\right)$ (в деформированном Ge в $1 s^{(3)}\left(\Gamma_{3}\right)$ ) при излучении ТА фононов с волновыми векторами в окрестности X точки зоны Бриллюэна происходят из высокоэнергетических состояний донора (главное квантовое число $n \geq 5$ ). Расчет роли этих переходов осложнен тем, что нет определенного состояния, из которого происходит переход. Переходы осуществляются из бесконечного набора состояний, лежащих в полосе энергий шириной $\sim 0.5$ мэВ под самым дном зоны проводимости. Однако можно сделать следующую оценку: вычислять темп перехода из различных состояний, огибающая волновых функций которых характеризуется различными квантовыми числами $n, l, m$ на переходе в состояние $1 s^{(3)}\left(\Gamma_{5}\right)$ (в деформированном Ge в $\left.1 s^{(3)}\left(\Gamma_{3}\right)\right)$, интерполировать полученные значения для случая больших $n$ и определить среднюю величину скорости перехода. Имеет смысл именно средняя в еличина, так как электрон в процессе релаксации не проходит через все состояния из этого пояска энергий, поскольку он слишком узкий. Электрон захватывается из зоны проводимости на любое из состояний в этом пояске, а затем следующим же актом излучения фонона (внутридолинного или междолинного) выходит из него. Максимальная вероятность перехода будет из состояний s-типа, переходы из других состояний (с квантовыми числами $p, f, h$ и т.д.) будут заметно медленнее. Это связано с тем, что матричный элемент перехода тем больше, чем ближе топологии волновых функций состояний перехода, поэтому переход с большей вероятностью происходит между состояниями с одинаковыми квантовыми числами $l$ и $m$, которые характеризуют огибающие состояний перехода. Поэтому при вычислении закономерности изменения вероятности при изменении $n$ начального состояния можно ограничиться рассмотрением переходов только между состояниями $s$-типа. Были вычислены темпы переходов в состояние $1 s^{(3)}\left(\Gamma_{5}\right)$ из $1 s, 2 s, 3 s, 4 s$ состояний при одинаковых резонансных условиях, которые довольно гладко ложатся на монотонно спадающую зависимость темпа от $n$ (рис. 4). При

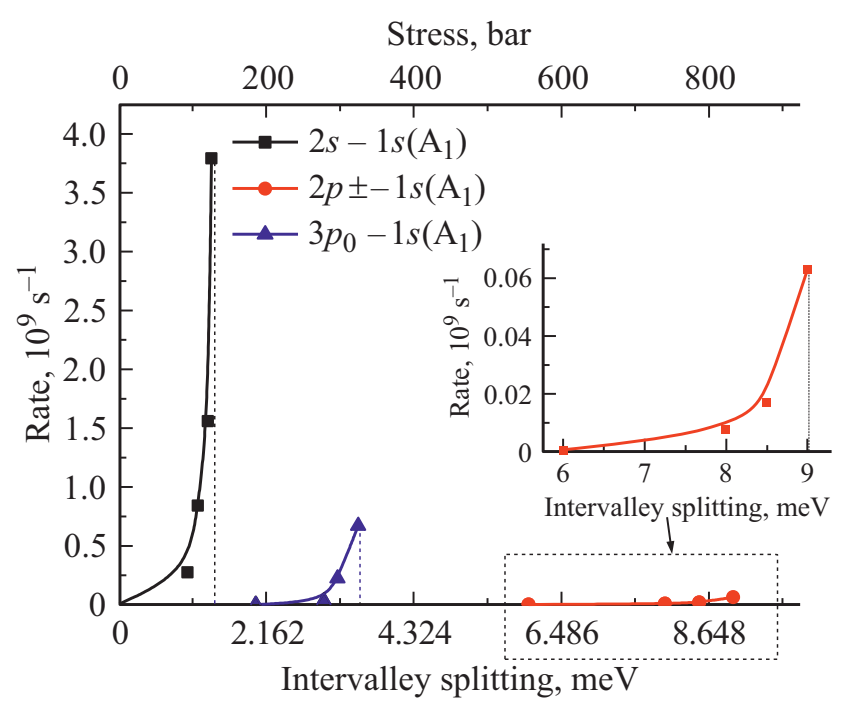

Рис. 3. Темпы некоторых основных междолинных внутрицентровых переходов в $\mathrm{Ge}: \mathrm{As}$ в зависимости от одноосной деформации сжатия кристалла в направлении $\{111\}$.

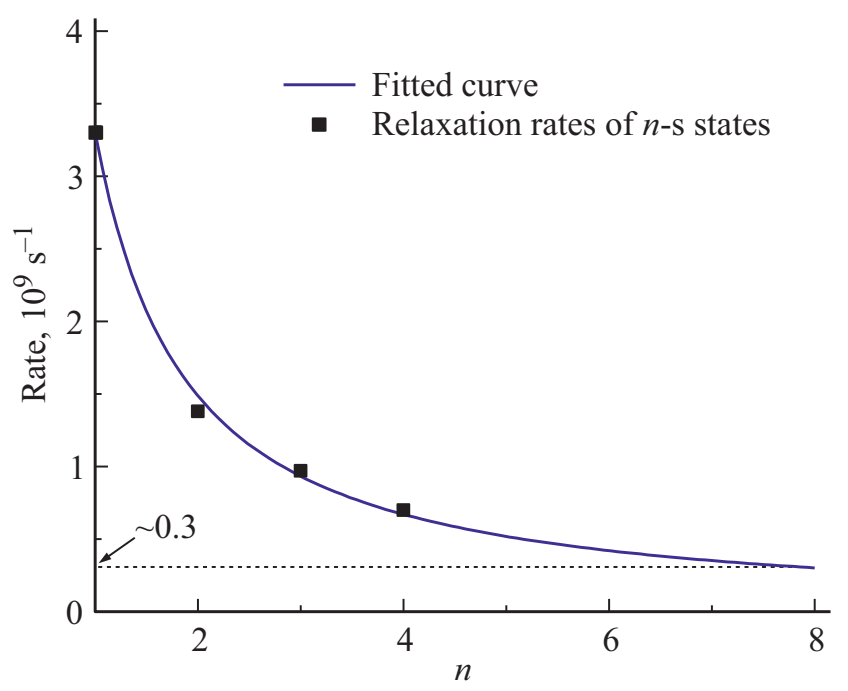

Рис. 4. Темпы междолинных переходов из $1 s, 2 s, 3 s, 4 s$ в состояние $1 s$ триплета при одинаковых резонансных условиях (энергия перехода 9.75 мэВ). 
достаточно больших $(n=5,6,7,8)$ темп начинает не очень сильно изменяться с изменением $n$, при этом его среднее значение имеет величину $\sim 0.3 \cdot 10^{9} \mathrm{c}^{-1}$, которую можно взять за оценку средней скорости междолинного перехода из рассматриваемой полосы состояний в состояние $1 s^{(3)}\left(\Gamma_{5}\right)$. В сопоставлении со значениями скоростей других переходов в $n$-Ge $[7,8]$, полученное значение темпа является довольно существенным.

\section{3. Заключение}

Согласно проведенным вычислениям, темпы междолинных переходов крайне сильно зависят от энергии внутрицентровых переходов. Вследствие этого зависимости темпов внутрицентровых междолинных переходов от одноосной деформации сжатия кристалла представляют собой острые пики с шириной всего 20-50 бар, достигающие величины $\sim 10^{9} \mathrm{c}^{-1}$. Средняя скорость междолинного перехода из состояний, находящихся в пояске энергий шириной $\sim 0.5$ мэВ под самым дном зоны проводимости, в состояния триплета $1 s(\mathrm{~T} 2)$ оценивается как $0.3 \cdot 10^{9} \mathrm{c}^{-1}$, что составляет довольно заметную величину на фоне других темпов внутрицентровых переходов в донорах мышьяка в германии. Полученные результаты можно перенести и на доноров сурьмы $(\mathrm{Sb})$ в германии. B $\mathrm{Ge}: \mathrm{Sb}$ роль таких процессов, по-видимому, должна быть более существенна, из-за того что энергетический зазор между состояниями $1 s(\mathrm{~A})$ и $1 s(\mathrm{~T})$ всего $\sim 0.5$ мэВ, и переходы из высоковозбужденных состояний должны происходить не только в состояние $1 s(\mathrm{~T})$, но и в основное состояние донора $1 s(\mathrm{~A})$.

Таким образом, при анализе экспериментальных результатов, pump-probe откликов при внутрицентровом оптическом возбуждении, должны приниматься во внимание междолинные процессы излучения фононов. Даже когда возбуждение осуществляется в состояния, находящиеся по энергии ниже пояска, из которого может осуществляться сброс в состояния $1 s$ триплета, нужно учитывать нагрев кристалла излучением лазера. Вследствие этого нагрева происходит быстрое размытие функции распределения электронов по всем окрестным состояниям, в том числе лежащих по энергии выше возбуждаемого уровня, из которых потом возможен сброс носителей сразу в возбужденные $1 s$ состояния триплета при междолинных переходах с излучением коротковолновых акустических фононов. В условиях же одноосной деформации кристалла релаксация может происходить при междолинных переходах сразу в основное состояние донора. Однако следует помнить, что в эксперименте сложно обеспечить однородность деформации исследуемого образца, и вряд ли в эксперименте будут наблюдаться столь резкие изменения релаксационных темпов состояний донора (в соответствии с результатами расчета на рис. 3) в зависимости от прикладываемого к кристаллу давления. Зависимости будут сглажены, и влияние междолинных переходов по сравнению с расчетом будет проявляться в немного меньшей степени, но в более широком диапазоне прикладываемых к исследуемому образцу величин давления.

\section{Финансирование работы}

Работа выполнена при поддержке Российского научного фонда (соглашение № 19-72-20163).

\section{Конфликт интересов}

Авторы заявляют, что у них нет конфликта интересов.

\section{Список литературы}

[1] A.P. Heberle, J.J. Baumberg, E. Binder, T. Kuhn, K. Köhler, K.H. Ploog. IEEE J. Select. Top. Quant. Electron., 2, 769 (1996).

[2] A.M. Stoneham, A.J. Fisher, P.T. Greenland. J. Phys. Condens. Matter, 15, L447 (2003).

[3] L.C.L. Hollenberg, C.J. Wellard, C.I. Pakes, A.G. Fowler. Phys. Rev. B, 69, 233301 (2004).

[4] J.J. Pla, K.Y. Tan, J.P. Dehollain, W.H. Lim, J.J.L. Morton, F.A. Zwanenburg, D.N. Jamieson, A.S. Dzurak, A. Morello. Nature, 496, 334 (2013).

[5] A.J. Sigillito, A.M. Tyryshkin, J.W. Beeman, E.E. Haller, K.M. Itoh, S.A. Lyon. Phys. Rev. B, 94, 125204 (2016).

[6] K.J. Morse, R.J.S. Abraham, A. DeAbreu, C. Bowness, T.S. Richards, H. Riemann, N.V. Abrosimov, P. Becker, HansJoachim Pohl, M.L.W. Thewalt, S. Simmons. Sci. Adv. /Quant. Phys., 3, e1700930 (2017).

[7] Р.Х. Жукавин, К.А. Ковалевский, С.М. Сергеев, Ю.Ю. Чопорова, В.В. Герасимов, В.В. Цыпленков, Б.А. Князев, Н.В. Абросимов, С.Г. Павлов, В.Н. Шастин, Г. Шнайдер, Н. Дессманн, О.А. Шевченко, Н.А. Винокуров, Г.Н. Кулипанов, Г.-В. Хьюберс. Письма ЖЭТФ, 106, 555 (2017)

[8] Р.Х. Жукавин, К.А. Ковалевский, Ю.Ю. Чопорова, В.В. Цыпленков, В.В. Герасимов, П.А. Бушуйкин, Б.А. Князев, Н.В. Абросимов, С.Г. Павлов, Г.-В. Хьюберс, В.Н. Шастин. Письма ЖЭТФ, 110, 677 (2019)

[9] W. Kohn, J.M. Luttinger. Phys. Rev., 90 (4), 915 (1955).

[10] R.J. Bell, W.T. Bousman Jr.G.M. Goldman, D.G. Rathbun. Surf. Sci., 7, 293 (1967).

[11] В.В. Цыпленков, В.Н. Шастин. ФТП, 53, 1372 (2019).

[12] Б.И. Шкловский, А.Л. Эфрос. Электронные свойства легированных полупроводников (М., Наука, 1979) гл. 4, с. 38.

[13] В.В. Цыпленков, Е.В. Демидов, К.А. Ковалевский, В.Н. Шастин. ФТП, 42, 1032 (2008).

[14] В.В. Цыпленков, К.А. Ковалевский, В.Н. Шастин. ФТП, 43, 1450 (2009).

[15] C. Jacoboni, L. Reggiani. Rev. Mod. Phys., 55 (3), 645 (1983).

[16] P. Giannozzi, S. de Gironcoli, P. Pavone, S. Baroni. Phys. Rev. B, 41, 7231 (1991)

[17] M. Gienger, P. Gross, K. Lassmann. Phys. Rev. Lett., 64, 1138 (1990).

[18] R.J. Baker, P. Fisher. Sol. St. Commun., 99 (10), 679 (1996).

Редактор Г.А. Оганесян 


\section{Intervalley relaxation processes of shallow donor states in germanium}

V.V. Tsyplenkov, V.N. Shastin

Institute for Physics of Microstructures

Russian Academy of Sciences,

607680 Nizhny Novgorod, Russia

Abstract The role of the intervalley processes of electronphonon interaction in the relaxation of excited shallow arsenic donors in germanium is analyzed. The rates of intracenter intervalley transitions with emission of TA phonons in germanium are calculated in dependence on the uniaxial compression stress along $\{111\}$ crystallographic direction. It is shown that intervalley transitions to the ground state of the donor with emission of phonons can play a significant role in the relaxation of excited impurities only upon uniaxial stress of the crystal, since at zero stress, there are no exact resonances between impurity transitions and intervalley phonons. There are also transitions from highly excited states lying in a narrow band of energies $(\sim 0.5 \mathrm{meV})$ under very bottom of the conduction band to the first excited state $1 s^{(3)}\left(\Gamma_{5}\right)$ (in stressed germanium crystal to $1 s^{(3)}\left(\right.$ Gamma $\left._{3}\right)$ state). The average rate of these transitions is estimated at $0.3 \cdot 10^{9} \mathrm{~s}^{-1}$. 